\title{
The Measurement of Magnetic Hysteresis in Rocks and Minerals at High Temperatures
}

\author{
By E. R. DEUTSCH \\ Physics Department, Imperial College of Sience and Technology, London, England.
}

(Received Oct. 1, 1956)

\begin{abstract}
An experimental method is described, designed chiefly to furnish data for a study of the direction, and stability with time, of thermoremanence in rocks. Specimens were heated to the Curie point in an evacuated electric furnace. Two pick-up coils were arranged close to the gap of a tuned a.c. electromagnet providing a maximum field $H$ of 2400 oersteds. These were balanced to make their resultant e.m.f. zero in the presence of $H$ alone, and proportional to $d I / d t$ when a specimen of intensity of magnetization $I$ was in the gap. This e.m.f. was applied to the vertical plates of a cathode ray oscilloscope. The potential drop over a small resistance in the electromagnet input was applied to the deflection coils, giving a measure of $H$. Computations based on the resulting pattern on the c.r.o. screen yield a loop of the $I-H$ type, with $I$ in arbitrary units, from which the coercivity can be evaluated. A pronounced "sawtooth" pattern has been observed in the $(d I / d t)-H$ traces of pyrrhotite and franklinite specimens, particularly just below the respective Curie points.
\end{abstract}

\section{The Continuous Recording of Hysteresis Curves}

Several investigations [1], [2], [3], [4] have been made into the magnetic hysteresis of rocks, generally by stepwise measurements using a ballistic arrangement or a magnetometer. This method is slow and particularly unsuited to high temperature work when it is difficult to maintain experimental conditions constant for long periods.

Cathode rays were used, first by Ångström [5] and later by others [6], [7], [8], [9] for the investigation of the hysteresis curves of strongly ferromagnetic materials. Bruckshaw and Rao [10] first used an oscilloscope to measure the hysteresis of rocks. Air-cored coils, driven by a generator at $500 \mathrm{c} / \mathrm{s}$, provided the field $H$. The e.m.f. across an assembly of balanced pick-up coils was zero in the absence of the specimen, and proportional to $d I / d t$ when a specimen of intensity of magnetization $I$ was introduced into one of the coils. This e.m.f. was applied to the vertical plates in the c.r.o. The potential drop across a small resistance in series with the input was applied to the horizontal plates, the resulting deflection providing a measure of $H$. A pattern of the $(d I / d t)-H$ type was then traced on the c.r.o. screen. A numerical 
integration of the vertical sweep was required to convert this into an $I-H$ loop. Igneous rocks were investigated at room temperature in maximum fields of 750 oersteds.

\section{Experimental Requirements}

It can be shown that the field required to saturate spherical grains of magnetite falls in the range of 2000 to 3000 oersteds at room temperature. For several other strongly ferromagnetic minerals the saturation field appears to be also of this order. The equipment should therefore be designed to provide a magnetizing field of 2000 to 3000 oersteds. This field, as well as the temperature, must be as nearly possible constant over the volume occupied by a standard specimen, i.e. 4 to $5 \mathrm{~cm}^{3}$ in the equipment described here.

The method must be capable of measuring the relatively small intensity of magnetization of a specimen, in the presence of the magnetizing field. For a standard specimen of incremental volume susceptibility $2000 \times 10^{-6}$ c.g.s. units, measured in a weak field, this intensity accounted for roughly one part in $20 \% 0$ of the total induction in the equipment employed.

To make observations up to the Curie points of the materials concerned, a maximum temperature of $600^{\circ} \mathrm{C}$ was necessary for the ferromagnetic minerals here examined, and this involved a satisfactory method of measuring the temperature. To prevent oxidation of the specimen in the laboratory, it must either be surrounded by an inert atmosphere, such as nitrogen or argon, or heated in a vacuum.

\section{Summary of the Method}

The method used by Bruckshaw and Rao, briefly described above, was modified for work at high temperatures. A tuned circuit at $50 \mathrm{c} / \mathrm{s}$ in which an electromagnet acted as the inductance now provided magnetizing fields up to 2400 oersteds. Two coils were placed near the air gap, the first being mounted on the core. The second coil, in an adjustable position outside the core, was threaded by part of the flux spreading outwards from the gap. These coils were balanced in such a way that the e.m.f.s set up in them by the magnetizing field alone, cancelled out.

The specimen was in a vacuum inside an electric furnace. With specimen and furnace in the gap, the alternating field magnetized the specimen, causing a negligible increase in the total flux of the magnetic circuit. Due to the respective positions of the pick-up coils in relation to the gap, a differential e.m.f. was produced, and was directly proportional to $d I / d t$ of the specimen.

This e.m.f., after suitable amplification, was applied to the vertical deflection plates of an oscilloscope. The potential drop along a small resistance in series with the electromagnet was applied to the horizontal deflection coils of the oscilloscope, giving a measure of $H$.

Again it was necessary to convert the vertical scale from $d I / d t$ to $I$, and these calculations were made on data obtained from an enlarged photograph of the 
pattern, yielding a hysteresis loop of the $I-H$ type, in which $I$ was in arbitrary units.

\section{The Magnetizing Field}

Electromagnet (Fig. 1)

To avoid eddy currents, the core was built up from suitably trimmed transformer laminations of "stalloy," a high-permeability silicon steel. $A$ $3 \mathrm{~cm}$ gap was left between the pole pieces, which were interleaved with the yoke to prevent breaks in the magnetic circuit.

Two magnetizing coils $M$, each of 1000 turns of no. 16 S.W.G. specially in-

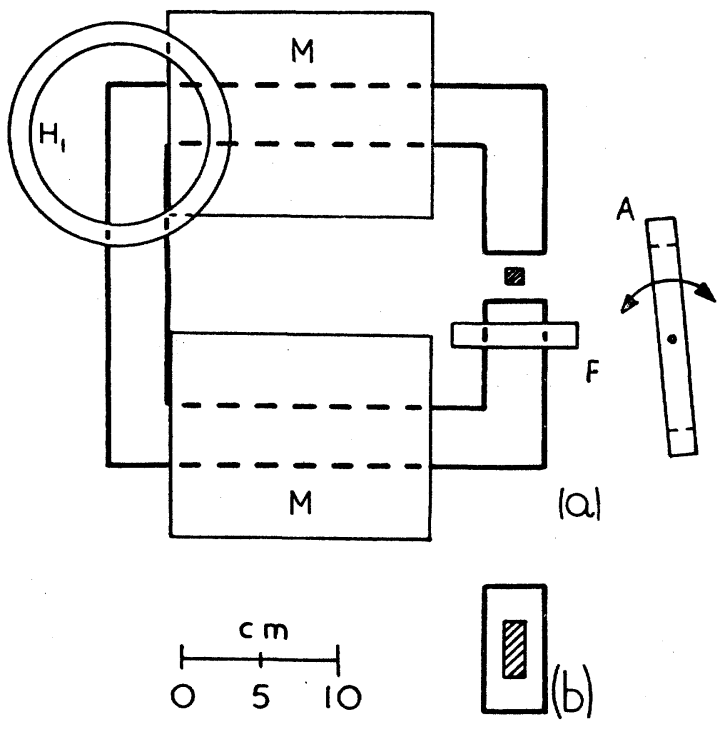

Fig. 1 sulated copper wire, were wound on Tufnol formers of rectangular cross-section to fit the yoke in the position indicated in Fig. 1. The complete electromagnet was assembled with Tufnol clamps and bolted to a wooden table in a horizontal position.

Tuning Circuit (Fig. 2)

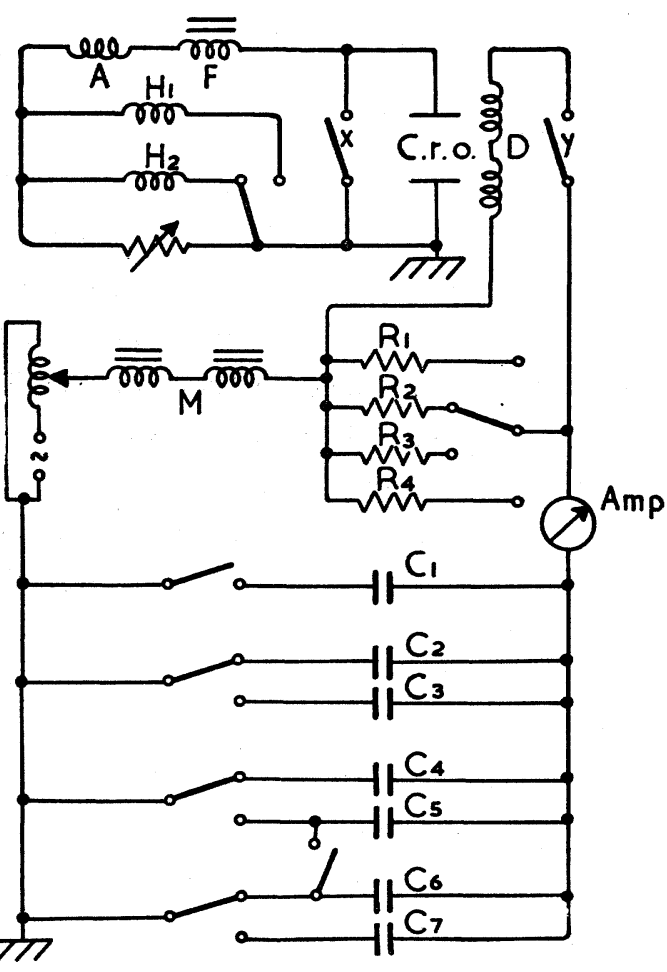

Fig. 2

The power for the magnetizing coils was obtained from the mains through an auto-transformer which supplied a maximum of $270 \mathrm{~V}$, insufficient to drive more than a small fraction of the required current through the coils of total impedance $720 \Omega$. The impedance was reduced by a condenser $C$ in series with the coils. Since the permeability of the core varied with the field, the self-inductance was a function of the magnetizing current, and a range of capacities was necessary to obtain different peak fields.

Since the gap was wide, the largest flux-density attained by the electromagnet was only $1700 G$, at 3 amp (both r.m.s. values), corresponding to a field of amplitude 2400 oersteds. All fields up to this maximum could be obtained by tuning the magnetizing coils with the range of capacities 3.7 
to $4 \cdot 3 \mu F$.

\section{Measurement of the Magnetizing Field}

A search coil whose effective area and shape were approximately those of a standard specimen could be positioned centrally in the gap. The reading on an a.c. voltmeter, multiplied by the appropriate factor, then gave the average field amplitude $H_{0}$ over the specimen volume.

A gap survey, carried out with a smaller search coil, revealed that the field in the volume occupied by a standard specimen varied by $\pm 2 \%$ from this average, the maximum field being found along the line joining the centres of the pole pieces.

\section{The Pick-up Circuit}

A rectangular former $F$ wound with 200 turns of no. 30 S.W.G. enamelled copper wire was fixed rigidly to one of the pole pieces, the coil centre being $3.7 \mathrm{~cm}$ from the gap centre.

The second, adjustable pick-up coil $A$, with 2500 circular turns of double silkcovered wire (40 S.W.G.) was placed close to the gap, to be threaded by some of the flux spreading outwards. As the ratio of the flux lines threading each of the two coils varied slightly for different magnetizing fields, the air-cored coil could be balanced by rotation with a fine adjustment control. Both formers and the adjusting mechanism were made of Tufnol.

\section{Harmonics Coils}

When the fundamental frequency was balanced a small e.m.f. remained, consisting at the lower fields chiefly of third harmonic. The relative positions of the two pick-up coils (Fig. 1) were chosen by trial to give a minimum of these harmonics.

A large coil $H 1$ of 2100 turns was mounted in a position (Fig. 1) where the resultant flux due to the field surrounding the two magnetizing coils was zero at the fundamental frequency. The e.m.f. set up in this coil then consisted mainly of third harmonic. After suitable adjustment of the amplitude by a potentiometer in the circuit, this was balanced against the harmonic residue in the pick-up coils, most of which was eliminated by this method. In fields above 1500 oersteds, a small coil $\mathrm{H}_{2}$ of 900 turns (Fig. 2) reduced the amplitude of the undesirable harmonics, but since these were now more complex, they balanced only to a minor degree. Both harmonics coils were made of double silk-covered copper wire (40 S.W.G.) wound on Tufnol formers whose position above the electromagnet was adjustable.

All the leads of the pick-up circuit (Fig. 2) were electrostatically screened.

\section{The Oscilloscope Pattern}

The oscilloscope was a Cossor double beam instrument giving a blue screen pattern.

\section{Vertical Deflection.}

The output of the pick-up system was fed into the c.r.o. amplifier, with a maximum gain of 900 , and the amplified e.m.f. applied to the vertical plates to the 
tube.

\section{Horizontal Deflection.}

The potential drop across a small resistance $R$ in series with the electromagnet was applied to the c.r.o. deflection coils $D$. The plates could not be used since both pairs, as well as the autotransformer, were earthed on one side. Depending on the input current, the tapping was made from one of a set of nichrome resistances in the range 0.3 to $1.7 \Omega$, selected to give full screen deflection.

By means of suitable switches $x$ and $y$ in the c.r.o. input, the coordinate axes could be superimposed on the photograph of each pattern.

\section{Deflection Calibration.}

The deflections on the tube screen were found to be proportional to the input, up to the dimensions of the pattern. These were generally confined within a central area of $5 \mathrm{~cm}$ diameter, where the screen was made flat to eliminate curvature error.

\section{Photography}

A Cossor $35 \mathrm{~mm}$ camera with manually operated shutter was used. Blue-sensitive Kodak $R 20$ and Ilford $5 \mathrm{Bll}$ films were selected, both types having high resolution. With low brilliance to render fine outlines on the curves, a suitable exposure for patterns was $\frac{3}{4} \mathrm{~s}$. The axes required much shorter timing.

\section{Furnace and Thermocouples}

The furnace (Fig. 3), essentially a long quartz tube wound with resistance wire, was of the general type used by Tomlinson and Bockris [11] in the range 1000 to $2000^{\circ} \mathrm{C}$. It was sufficiently narrow to fit between the pole pieces, leaving an air cushion 2 to $3 \mathrm{~mm}$ wide on each side.

A short, perforated quartz tube, fused inside to the bottom of the furnace, supported a quartz crucible containing the specimen. Heat conduction away from the crucible was thus greatly reduced, whilst convection was overcome by evacuating the tube through an outlet. A ground pyrex cone sealed the furnace vacuum-tight at one end, and, since the vacuum grease melted after slight heating, the furnace top was water cooled. Tungsten rods sealed through the pyrex provided contacts for the thermocouple.

The furnace was wound with 43 turns of no. 27 S.W.G. "nonmagnetic"

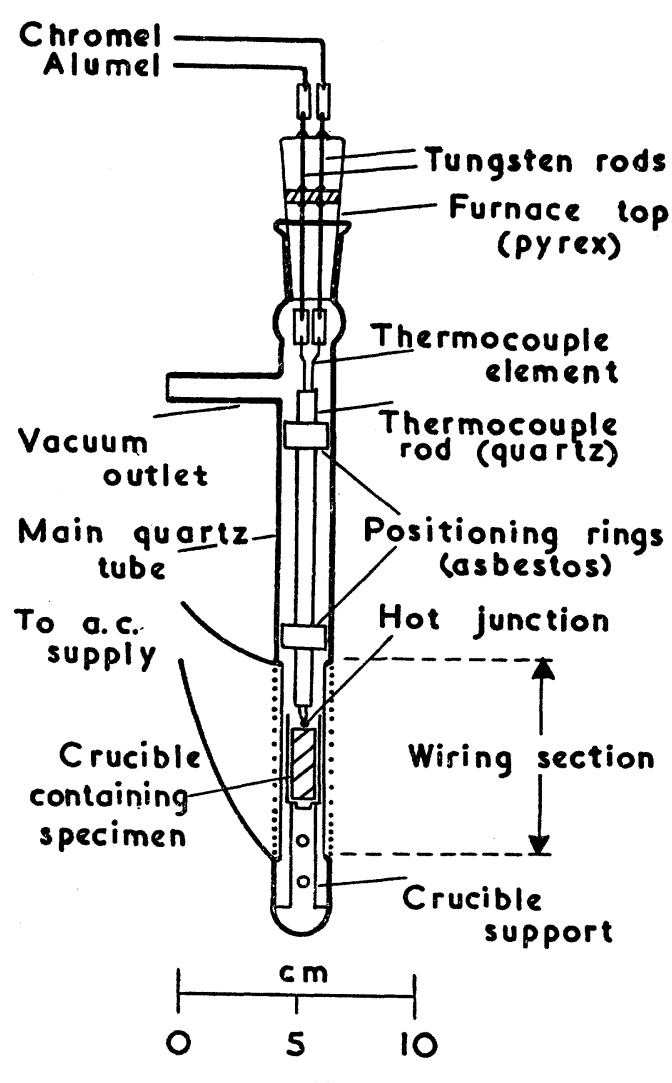

Fig. 3 
nichrome wire, concentrated at the top and bottom. With this arrangement, the temperature in the region normally occupied by a specimen was found to vary by $\pm 1 \%$ from its average value. A sheet of mica surrounding the wiring section, together with the air cushion, provided partial heat insulation for the pole pieces. Since, at high temperatures, the tube was left in the gap for at most $15 \mathrm{~s}$., further insulation was found unnecessary. The a.c. supply from an auto-transformer heated a specimen to $600^{\circ} \mathrm{C}$ in $10 \mathrm{~min}$, with $2.75 \mathrm{amp}$, but no advantage was gained from heating so quickly. To prevent induction in the pick-up coils from the heating coil, the supply was cut off for a short interval during the photographic exposure. During this interval the specimen temperature dropped by about $1^{\circ} \mathrm{C}$ at $600^{\circ} \mathrm{C}$, and this was neglected.

The furnace was kept in a raised position until the required temperature was reached, and then lowered into the gap by means of a brass ratchet arrangement.

\section{Thermocouple Circuit}

A chromel-alumel element yielding a large thermal e.m.f. was used. The junction was arc-welded into a bead, in a reducing atmosphere. To diminish the magnetic effect caused below $140^{\circ} \mathrm{C}$ by the nickel in chromel and alumel, the thermocouples were made of no. 26 S.W.G. wire, the smallest gauge available at the time. Any remaining magnetic background was accounted for by subtraction of the oscillogram pattern due to the thermocouple alone, from that obtained jointly with specimen and thermocouple. This precaution was only necessary with a few relatively weakly magnetic specimens. The hot junction was positioned centrally inside the furnace (Fig. 3).

A chromel-alumel cold junction was placed in ice inside a Dewar flask. Readings were taken on a galvanometer whose full deflection corresponded to about $60^{\circ} \mathrm{C}$, the higher temperature ranges being obtained by using suitable resistances in the circuit. To prevent secondary thermal e.m.f.s., all the wire in the thermocouple circuit was thick chromel or alumel. Pairs of metal contacts at the galvanometer, furnace top, cold junction head and switches, respectively, were closely spaced to equalize the temperature of each pair.

\section{Accuracy of Temperature Measurements}

A standard platinum-platinum/rhodium couple was used to calibrate two chromelalumel elements. The latter gave readings differing by up to $2^{\circ} \mathrm{C}$ at $600^{\circ} \mathrm{C}$, where the temperature could be read to $1^{\circ} \mathrm{C}$. Together with smaller errors, due to local heating at contacts, lag in the galvanometer response, etc., the uncertainty in the temperature determination may have amounted to $\pm 5^{\circ} \mathrm{C}$ at $600^{\circ} \mathrm{C}$.

\section{The Vacuum System}

The furnace was evacuated by means of a two-stage pump, the vacuum circuit being thick-walled rubber tubing sealed to glass connections with wax. The vacuum, measured with a mercury level gauge, averaged about $1 \mathrm{~mm}$ of mercury, sufficient to prevent any significant oxidation of specimen or thermocouple in the temperature range used. 


\section{The Specimens}

Specimens investigated included the strongly ferromagnetic minerals magnetite, pyrrhotite and franklinite, as well as rocks such as basalts, in which magnetite or titanomagnetite commonly occur as minor constituents. Specimens were cut into prisms $3.0 \mathrm{~cm}$ long and $1.1 \times 1.1 \mathrm{~cm}$ in cross-section. When powder concentrations of a ferromagnetic mineral were required for study, the mineral powder was mixed with plaster of Paris powder. Water was added and the resulting paste allowed to set in a mould. Artificial " rock" specimens were thus produced.

\section{Computation of the Hysteresis Curves}

If $H$ denotes the field in the magnetic circuit and $B$ the induction, then $H=B$ in the air gap. A specimen sets up further lines of induction, such that

$$
B=H+4 \pi I
$$

where $I$ is its intensity of magnetization. Since $B, H$ and $I$ are vectors, this relation is numerically exact only for specimens saturated in a uniform field. Using an a.c. electromagnet, the e.m.f. set up in a pick-up coil is then proportional to $d B / d t$, where

$$
d B / d t=d H / d t+4 \pi(d I / d t) .
$$

Since $d H / d t$ is balanced out in the method used, the field term vanishes and the net e.m.f. becomes proportional to $d I / d t$ only. For a sinusoidal input current of frequency $f$

$$
H=H_{0} \sin \omega t
$$

where $H_{0}$ is the peak field and $\omega=2 \pi f$.

So

$$
d H / d t=H_{0} \omega \cos \omega t
$$

and since

$$
\begin{aligned}
& d I / d t=(d I / d H) \times(d H / d t) \\
& d I / d t \propto(d I / d H) \cos \omega t .
\end{aligned}
$$

An ideal pattern on the c.r.o. screen is then proportional to expression (3) horizontally and to (6) vertically. In practice, the length of the horizontal trace increased linearly with $H$ up to nearly 1200 oersteds, but at higher fields this increase became larger as $H$ was raised, and it was necessary to calibrate the $x$-axis.

A calibration chart contained the coordinate axes as well as vertical lines intersecting the abscissa at intervals corresponding to steps of $0.05 H_{0}$. This was accurately superimposed on a projected oscillogram of the pattern, whose height could then be measured directly at regular field intervals. A typical

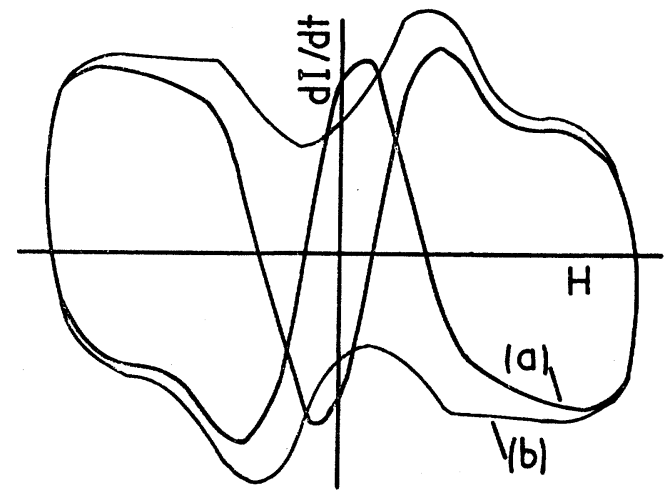

Fig. 4 
oscillogram, taken at $H_{0}=2300$ oersteds, has been retraced in Fig. 4. At each interval, the difference between the main lcop (a) and loop corresponding to unbalanced harmonics (b) was measured, giving net readings caused by the specimen alone. Values proportional to $d I / d t$ thus obtained along the ordinate were converted into quantities proportional to $d I / d H$ after division by $\cos \omega t$ (Fig. 5). A numerical integration between limits of $0.05 \mathrm{H}_{0}$, proceeding from the origin at an arbitrary abscissa, then yielded the

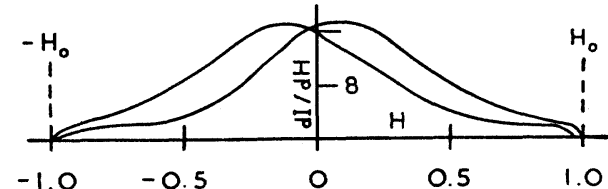

Fig. 5 desired $I-H$ curve (Fig. 6a).

Half the vertical separation between the maximum and minimum of $I$ was taken as the saturation magnetization $I_{s}$. Half the separation between the intercepts at $H=0$ gave the remanence $I_{r}$. The horizontal axis could be obtained by locating a point along the ordinate axis either halfway between $+I_{s}$ and $-I_{s}$, or halfway between $+I_{r}$ and $-I_{r}$. In an ideal hysteresis loop these points should coincide at the true origin, but due to experimental errors involving

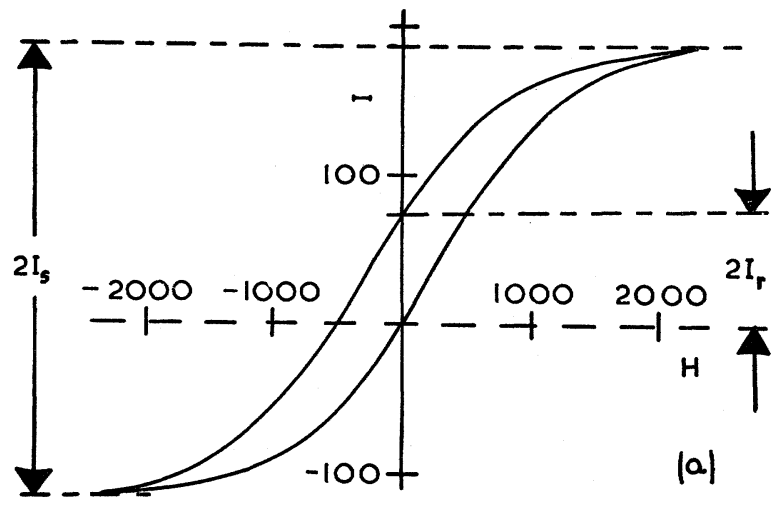

Fig. 6a mainly the oscilloscope measurements, the loop may be slightly distorted and fail to close. When this has been the case (Fig. 6b), the final abscissa was drawn through an origin located in a mean position along the ordinate. Half the separation between the intercepts on the abscissa then yielded the coercivity $H_{r}$.

From the distortion in the quoted example, $H_{c}, I_{r}$ and $I_{s}$ were estimated to be accurate to $\pm 1.6,2.5$ and $0.6 \%$ respectively. These may be regarded as typical figures for strongly magnetic rocks. In smaller fields, involving simpler patterns, these errors decrease, whilst measurements at high temperatures or with weaker rocks entail correspondingly larger errors. Just below the Curie point, the $I-H$ loop tends to become very flat, and then the expected error in measuring $H_{c}$ may be as large as 10 to $20 \%$.

\section{The "Sawtooth" Pattern}

In Fig. $7(\mathrm{a}-\mathrm{f})$, oscillograms are reproduced for a pyrrhotite specimen $(P 1)$ at $H_{0}=1200$ oersteds and temperatures between $20^{\circ} \mathrm{C}$ and $310^{\circ} \mathrm{C}$. To

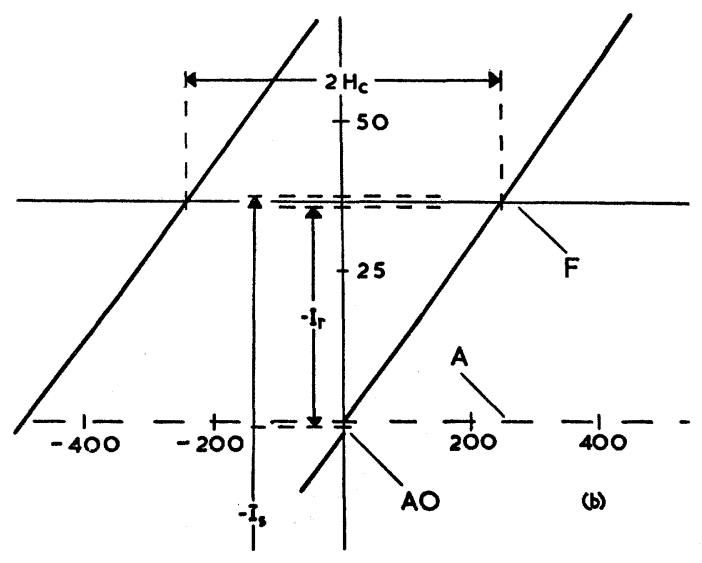

Fig. 6b 
facilitate the computing, the harmonics loop was expanded to surround the main pattern, which remained relatively unchanged up to $210^{\circ} \mathrm{C}$, but rapidly altered between $295^{\circ} \mathrm{C}$ and $310^{\circ} \mathrm{C}$, corresponding to the collapse of the $I-H$ loop just below the Curie point.

At high temperatures, the oscillograms exhibited the "sawtooth" pattern (Fig. 7 (c-f)), which was most pronounced in the region of the coercive field and affected those parts of the loop where magnetization changes are likely to proceed chiefly through irreversible domain movements. The pattern resembled a damped oscillation with a regular period.

A phenomenon possibly analogous to this has been observed by Cross [12] in the neighbourhood of the electrical coercive field of barium titanate crystals, where a "kinking" occurred at a certain temperature below the Curie point. In the present case, however, the "sawtooth" pattern was not noticeable in the computed $I-H$ loops, except when they were greatly enlarged.

Three other examples of the "sawtooth" pattern, retraced in Fig. $8(\mathrm{a}-\mathrm{c})$, appeared to be most pronounced in the range $15^{\circ} \mathrm{C}$ to $50^{\circ} \mathrm{C}$ below the Curie points concerned (Table I).

Results of hysteresis measurements on a number of specimens are discussed in a separate paper in this issue of the Journal.
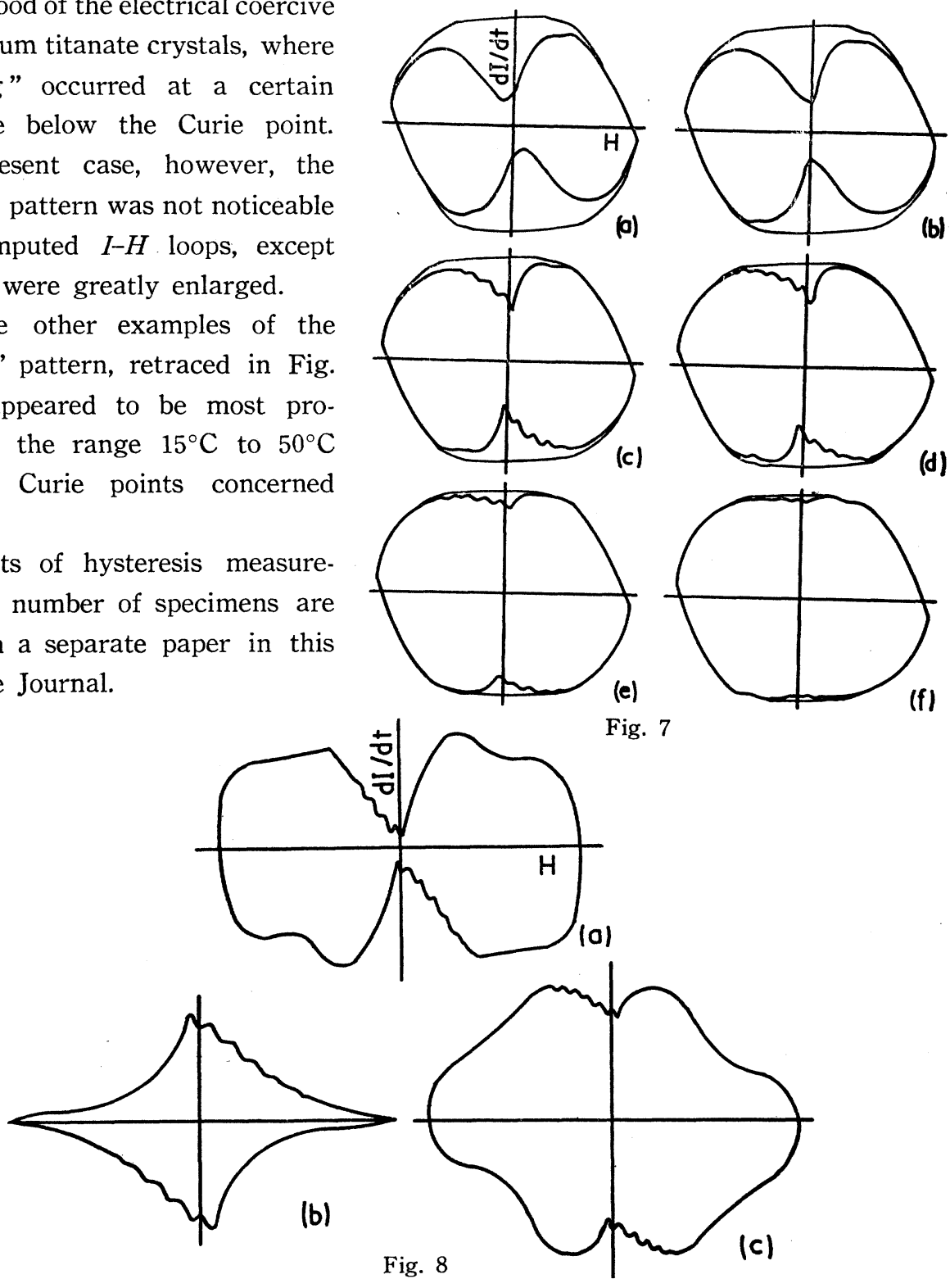

Fig. 7

(f) 
Table I. Temperature of pronounced "Sawtooth" patterns

\begin{tabular}{|c|c|c|c|}
\hline Specimen & $T_{c}\left({ }^{\circ} \mathrm{C}\right)$ & $T_{s}\left({ }^{\circ} \mathrm{C}\right)$ & $T_{c}-T_{s}\left({ }^{\circ} \mathrm{C}\right)$ \\
\hline$P 1$ (Pyrrhotite) & 330 & $305-300$ & $25-30$ \\
\hline$P 2$ (Pyrrhotite) & 320 & $295-270$ & $25-50$ \\
\hline$F 4$ (Franklinite) & 20 & 5 & 15 \\
\hline$F 5$ (Franklinite) & 525 & 480 & 45 \\
\hline
\end{tabular}

$T_{c}=$ Curie point.

$T_{s}=$ Temperature(s) at which pattern was photographed.

\section{Acknowledgments}

The author wishes to express his gratitude to Prof. J.M. Bruckshaw, $F$. Inst. $P$., for his help and encouragement throughout the work. Thanks are also due to the Anglo-Iranian Oil Company and the Anglo-Saxon Oil Company for providing the financial basis for this research.

\section{References}

[1] Nagata, T. Rock Magnetism, p. 105 (Tokyo : Maruzen and Co., Ltd., 1953).

[2] Puzicha, K. Z. Prakt. Geol. 38, 161, 184 (1930).

[3] Turcev, A. Bull. Acad. Sci. U.S.S.R. (Leningrad), Ser. 7, Phys.-Math. Sci., 89 (1928).

[4] Tzu-Chang-Wang. Z. Geophys. 16, 160 (1940).

[5] Ångström, K. Phys. Z. 1, 121 (1899); Phys. Rev. 10, 74 (1900).

[6] Cosens, C.R. Wireless Engr. 12, 190 (1935).

[7] Johnson, J.B. Bell Syst. Tech. J. 8, 286 (1929).

[8] Kreiselsheimer, K. J. Sci. Instrum. 19, 137 (1942).

[9] Madelung, E. Phys. Z. 8, 72 (1907).

[10] Bruckshaw, J.M. and Rao, B.S. Proc. Phys. Soc. (London) 63, 931 (1950).

[11] Tomlinson, J.W. and Bockris, J. O'M. Rev. Sci. Instrum. 21, 507 (1950).

[12] Cross, L.E. Phil. Mag. 44, 1161 (1953). 